\section{Evaluación del cuestionario SF-12}

\section{An appraisal of the SF-12 Questionnaire}

\section{Sr. Editor:}

En una reciente publicación se evaluaron las propiedades psicométricas del instrumento SF$12^{1}$, y para dicho fin los autores determinaron la validez de constructo mediante la evaluación de su estructura interna, usando un conjunto de procedimientos frecuentemente aplicados en la práctica del análisis factorial exploratorio ${ }^{2,3}$, pero a la vez la menos recomendada: análisis por componentes principales, extracción del número de factores a través de la regla del autovalor mayor a 1 y rotación ortogonal varimax.

Los autores afirman que, de acuerdo a sus resultados, el SF-12 es una escala que permite hacer medidas válidas del sujeto evaluado, y con el cual obtener un perfil del estado de salud físico, social y mental; pero esta afirmación creemos que puede tener un respaldo empírico cuestionable debido a que está reportado en la literatura metodológica que la regla del autovalor mayor a 1 es imprecisa y que produce una conocida tendencia a la sobreestimación en la elección del número apropiado de factores o componentes subyacentes a un conjunto de datos ${ }^{2-5}$.

En la literatura científica existe consenso al indicar que la determinación del número de factores es el paso más crítico e importante en un análisis factorial exploratorio, en el que la estructura interna de un instrumento psicométrico se establece mediante el número de factores que modela matemáticamente los datos obtenidos, y que en consecuencia determina la explicación conceptual del contenido investigado ${ }^{2}$. Por ello la utilización de la regla del autovalor mayor a 1 , al no brindar con precisión el número de factores, aumenta la probabilidad de que el investigador interprete factores meramente aleatorios, que pueden ser producto del error de medición, idiosincrásicos a la muestra, no replicables, sin sentido teórico y que no aportan a la interpretación del constructo. Por lo tanto, la aplicación de la regla del autovalor mayor a 1 puede invalidar parcialmente la inferencia que el investigador realice en base a las puntuaciones obtenidas de un instrumento. Es importante recalcarlo porque la aplicación del SF-12 está destinada principalmente al ámbito clínico, donde toda inferencia tiene incidencia directa en la persona.

Generalmente, la aplicación de la regla del autovalor mayor a 1 ha formado parte del otro conjunto de decisiones que involucraba el uso del análisis de componentes principales y la rotación varimax. En la historia del desarrollo del análisis de la dimensionalidad se ha denominado a este conjunto como "Little Jiffy". Este conjunto de procedimientos se aplicaba mediante un paquete informático (que usaba el mismo nombre) orientado hacia la identificación de dimensiones ortogonales (estadísticamente independientes entre sí) y obtención de soluciones factoriales simples (es decir, los ítems mostraban la mayor carga posible en su propio componente, y cargas cerca de cero en otros componentes). Sin embargo, los avances en los conocimientos científicos también van acompañados con progresos en las metodologías y, actualmente, el "Little Jiffy" no es recomendado en la búsqueda de la dimensionalidad de los instrumentos ${ }^{3-6-7}$. Si este enfoque es razonable para los datos analizados por Vera-Villarroel y colaboradores, entonces deben hacer una fuerte justificación de su elección metodológica, argumentación que no apareció en su trabajo.

Consideramos encomiable el esfuerzo realizado por los autores, pero ante el panorama metodológico actual y las exigencias metodológicas para que las pruebas psicométricas deban ser analizadas con métodos más apropiados, creemos que es necesario la realización de posteriores estudios donde se determine la estructura interna del SF12 utilizando metodologías recomendadas para la extracción del número de factores (por ejemplo, el análisis paralelo), la obtención de la solución factorial (por ejemplo, el enfoque de factores comunes) y la rotación de ésta última para mejorar la interpretación conceptual (por ejemplo, rotaciones oblicuas que permiten la correlación entre las variables latentes o factores) ${ }^{7}$.

\section{Jhonatan S. Navarro-Lolia, César Merino Soto ${ }^{b}$}

${ }^{a}$ Psicólogo, docente-investigador en el Instituto de Investigación de Psicología, Universidad de San Martín de Porres (Lima, Perú).

${ }^{b}$ Psicólogo, docente-investigador en el Instituto de Investigación de Psicología, Universidad de San Martín de Porres (Lima, Perú). 


\section{Referencias}

1. Vera-Villarroel P, Silva J, Celis-Atenas, K, Paves P. Evaluación del cuestionario SF-12: Verificación de la escala salud mental. Rev Med Chile 2014; 142: 127583.

2. Zwick W, Velicer W. Comparison of five rules for determining the number of components to retain. Psychol Bull 1986; 99: 432-42.

3. Fabrigar L, Wegener D, MacCallum R, Strahan E. Evaluating the use of exploratory factor analysis in psychological research. Psychol Methods 1999; 3: 272-99.

4. Hakstian R, Rogers T, Cattell B. The behavior of number of factor rules with simulated data. Multivar Beha Res 1982; 17: 193-219.

La carta fue enviada a los autores aludidos, quienes ofrecen la siguiente réplica:

\section{Evaluación del cuestionario SF-12 (Réplica)}

\section{An appraisal of the SF12 Questionnaire (Reply)}

\section{Sr. Editor:}

En respuesta a lo expuesto por J Navarro-Loli y C Merino Soto, exponemos lo siguiente:

Los autores ponen de relieve una temática de actual discusión en psicometría, la cual ha tenido mayor fuerza al ser socializada en las áreas metodológicas de la ciencia, no estando suficientemente difundida a toda la comunidad científica ${ }^{1,2}$. Desde esta controversia en relación a los métodos para evaluar la estructura interna de un instrumento, podemos concordar con los comentarios metodológicos propuestos, pero dejamos en claro que al momento del análisis métrico de un instrumento de medida no sólo se deben considerar los elementos metodológicos, sino que también los elementos constitutivos del constructo a medir y cómo este ha sido analizado en estudios previos y tradicionales con el fin de responder a la reproducibilidad en la investigación científica y en ciencia aplicada, criterio con el cual nosotros basamos nuestras decisiones para este artículo.

Nuestra toma de decisiones estuvo desde un enfoque clásico, donde el uso de extracción de factores se basó en componentes principales, considerando estudios en que el análisis factorial
5. Navarro J, Merino C. Un Réquiem para la regla de Keiser (eigen $>1$ ) para la retención del número de factores. Fractal: Revista de Psicología 2010; 22 (3): 640.

6. Preacher KJ, MacCallum RC. Repairing the Tom Swift's electric factor analysis machine. Understanding Statistics 2003; 2: 13-43.

7. Hayton J, Allen D, Scarpell V. Factor retention decisions in exploratory factor analysis: A tutorial on parallel analysis. Organ Res Methods 2004; 7: 191-205.

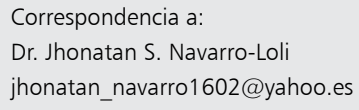

y el análisis de componentes principales son considerados como equivalentes, al igual que en el programa estadístico usado para los análisis de este articulo; por lo tanto, se basan en reglas como las de $\mathrm{K} 1^{3-4}$. Así mismo, la selección del método de rotación donde se privilegió el criterio teórico, es decir, donde se expresa la independencia de los factores propuestos originalmente ${ }^{5-7}$.

La elección de indicadores estuvo basada también en los métodos utilizados en adaptaciones previas al momento de realizar búsqueda bibliográfica, donde se evalúa la validez de constructo por medio de análisis factorial utilizando iguales criterios de extracción de factores y de rotación de estos ${ }^{8-10}$.

El interés principal fue poner foco en el análisis de la dimensión mental del instrumento, dado que tradicionalmente las publicaciones relacionadas con este instrumento se basan en muestras clínicas con patologías físicas de curso y tratamientos complejos. Para ello se integra el análisis de indicadores de validez externa, tal como se realiza en el segundo estudio, y como a nivel investigativo en áreas de salud, calidad de vida y bienestar desde la perspectiva psicológica, el cuestionario SF-12 tendría potencial al momento de evaluar la calidad de vida relacionada con la salud de las personas.

Tal como se propone en la discusión de nuestro artículo, es necesario aportar con mayor evidencia en cuanto a las propiedades del cuestionario SF12 tanto para la investigación como en ámbitos sanitarios donde se hace énfasis a la promoción 
de la salud, por tanto es de interés contar con herramientas precisas y válidas al momento de evaluar de forma subjetiva el estado de salud no tan sólo físico sino también mental.

El artículo de Vera-Villarroel et al (2014) ${ }^{11}$ contribuye al avance del conocimiento científico al mostrar evidencias empíricas del instrumento, tal como se ha realizado en estudios anteriores y aportando evidencia a uno de los aspectos no medidos tradicionalmente. Lo sugerido por $\mathbf{J}$ Navarro-Loli y C Merino Soto desde la experticia metodológica también contribuye a mejorar la precisión de los instrumentos, como el SF-12 y cualquier otro que contenga previamente una evidencia empírica tradicional.

Pablo Vera-Villarroel Universidad de Santiago de Chile

\section{Referencias}

1. Lloret-Segura S, Ferreres-Traver A, Hernández-Baeza A, Tomás-Marco I. El análisis factorial exploratorio de los ítems: una guía práctica, revisada y actualizada. Anal Psicol 2014; 30 (3): 1151-69. Doi: http://dx.doi. org/10.6018/analesps.30.3.199361.

2. Ferrando P, Lorenzo-Seva U. El análisis factorial exploratorio de los ítems: algunas consideraciones adicionales. Anal Psicol 2014: 30 (3): 1170-5. Doi: http://dx.doi. org/10.6018/analesps.30.3.199991.

3. Thompson B. (2004). Exploratory and confirmatory factor analysis: Understanding concepts and applications. Washington, DC: American Psychological Association.

4. Velicer WF, Jackson DN. Component analysis versus common factor analysis: Some issues in selecting an appropriate procedure. Multivariate Behavioral Res 1990; 25 (1): 1-28.

5. Monteagudo-Piqueras O, Hernando-Arizaleta L, Palomar-Rodríguez J. Normas poblacionales de referencia de la versión española del SF-12v2 para la Región de Murcia. Gac Sanit 2011; 25 (1): 50-61.

6. Rebollo P. Utilización del «grupo $\mathrm{SF}$ » de los cuestionarios de calidad de vida (SF-36, SF-12 y SF-6D) en el marco de ensayos clínicos y en la práctica clínica habitual. Med Clin (Barc) 2008; 130 (19): 740-1.

7. Tuesca-Molina R. La calidad de vida, su importancia y cómo medirla. Salud Uninorte. Barranquilla (Col.) 2005; 21: 76-86.

8. Wang W, López V, Sek Ying Ch, Thompson D. The psychometric properties of the Chinese version of the SF-36 health survey in patients with myocardial infarction in mainland China. Qual Life Res 2006; 15: 1525-31.

9. Taft C, Karlsson J, Sullivan M. Performance of the Swedish SF-36 version 2.0. Qual Life Res 2004; 13: 251-6.

10. Montazeri A, Vahdaninia M, Javad Mousavi S, Omidvari $S$. The Iranian version of 12 -item short form health survey (SF-12): Factor structure, internal consistency and construct validity. BMC Public Health 2009; 9: 341-51.

11. Vera-Villarroel P, Silva J, Celis-Atenas K, Pavez P. Evaluación del cuestionario SF-12: verificación de la utilidad de la escala salud mental. Rev Med Chile 2014; 142 (10): 1275-83.

Correspondencia a:

Pablo Vera-Villarroel pablo.vera@usach.cl 